\title{
PAYSAGES MEDITERRANEENS
}

\author{
MICHEL DRAIN ${ }^{1}$
}

Résumé - Les paysages méditerranéens dont l'extrême diversité ne peut masquer la profonde unité, sont particulièrement menacés de nos jours. Cela tient à l'arrivée soudaine et brutale de techniques venues d'ailleurs qui se sont substituées tardivement à des savoirfaire millénaires dans le cadre d'un milieu écologiquement fragile.

S'il convient de laisser les paysages évoluer et d'éviter de les brider à l'excès sous l'effet d'une conception par trop patrimoniale, il faut aussi éviter leur destruction. C'est bien le cas des paysages méditerranéens qui sont un véritable palimpseste où est inscrite l'histoire de la formation de notre identité territoriale.

On préconise donc à leur égard une évolution adaptée aux spécificités méditerranéennes ce qui est loin d'être le cas. On notera notamment qu'une partie des bouleversements des temps présents sont des effets de domination économique au profit des pays les plus développés. Ils incluent d'ailleurs, paradoxalement, une volonté de figer à usage externe des fragments idéalisés de paysages méditerranéens. A cet égard, le développement durable pourrait bien passer d'abord par un nouvel ordre international plus équilibré et plus juste.

Mots-clés: Méditerranée, paysages méditerranéens, évolution des paysages.

Resumo - PAISAGENS MEDITERRÂNEAS - As paisagens mediterrâneas, cuja extrema diversidade não pode ocultar a sua profunda unidade, estão, hoje em dia, particularmente ameaçadas. Este facto deve-se à difusão repentina e brutal de técnicas importadas, que se substituiram às práticas ancestrais num meio ecologicamente frágil.

Se, por um lado, se deve permitir a evolução das paisagens, evitando restringir o seu desenvolvimento, devido a uma concepção demasiado patrimonial, por outro lado, é necessário evitar a sua destruição. É o caso das paisagens mediterrâneas, nas quais está inscrita a história da formação da nossa identidade territorial.

Preconiza-se uma evolução adaptada às especificidades mediterrâneas, o que está longe de ocorrer. Notar-se-á que uma parte das perturbações actuais representam uma consequência do domínio económico pelos países mais desenvolvidos. Aliás estes têm, paradoxalmente, um desejo de conservar inalterados alguns fragmentos de paisagens mediterrâneas para «uso externo». Neste caso, o desenvolvimento sustentável deveria passar primeiro por uma nova ordem internacional mais equilibrada e mais justa.

1 Directeur de Recherche au C.N.R.S., Université de la Méditerranée. Fac. des Sciences de Luminy. Dep. Sciences Humaines. Case 901, 163, Av. de Luminy. 13288 Marseille Cedex 9 - France 
Palavras-chave: Mediterrâneo, paisagens mediterrâneas, evolução das paisagens, desenvolvimento sustentável.

\section{I - UNITE ET DIVERSITE DES PAYSAGES MEDITERRANEENS}

Il est d'usage d'enfermer le domaine méditerranéen dans les limites de la culture de l'olivier, bel exemple de syncrétisme entre nature et culture. Il répond à un climat spécifique dans lequel, cas unique au monde, la saison sèche coïncide avec la chaleur estivale et en constitue le seul trait immuable. Les conséquences biologiques en sont considérables. A la saison froide des climats tempérés, s'ajoute, en effet, la saison sèche des climats tropicaux qui ralentit la croissance des végétaux, les fragilise et, par voie de conséquence, expose les sols au lessivage et à l'érosion. Ces traits que l'on retrouve aux mêmes latitudes sur les façades occidentales des continents et, notamment, en Californie, prennent avec la Méditerranée une extension remarquable dans l'espace et le temps. La mer allongée d'est en ouest correspond à une gigantesque déchirure de l'écorce terrestre. Les deux bords qui se resserrent sont à l'origine d'un relief montagneux qui exacerbe un peu plus l'érosion linéaire. L'affrontement des plaques continentales s'exprime aussi sous forme de volcanisme et de séismes. Cette situation concourt à l'évolution rapide des paysages «naturels», y compris à l'échelle historique.

Si le paysage méditerranéen est bien ancré dans cette mise en scène grandiose que lui confèrent sa lumière, son décor minéral et la netteté de son architecture, il est également, depuis des millénaires, façonné par les hommes qui l'ont édifié et, plus tard, valorisé par le regard que l'on porte sur lui. Une longue et brillante histoire donne la dimension culturelle de cette portion de l'espace terrestre proche de quelques uns des plus anciens foyers de civilisation du monde. Dans un tel milieu d'ailleurs, les successions de vaches grasses et de vaches maigres étaient plus durement ressenties que sous un climat océanique par les collectivités paysannes conduites à rechercher des palliatifs à la sécheresse du côté de pratiques spécifiques telles que la transhumance ou le nomadisme, les cultures arbustives et l'agriculture irriguée. De là aussi cette diversité dont une récente étude portant sur la région française Provence-Côte d'Azur donne une idée avec pas moins de 84 unités paysagères sans prendre en compte pour autant les paysages urbains. Cette extraordinaire diversité qui faisait écrire à BRAUDEL (1977) que la Méditerranée était «Mille chose à la fois. Non pas un paysage mais d'innombrables paysages» s'intègre cependant dans un tout dont le même auteur écrivait qu'il constituait «une image cohérente, comme un système où tout se mélange et se recompose en une unité originale» ${ }^{2}$.

2 Braudel, F. (1977) La Méditerranée, L'espace et l'histoire. Paris. 


\section{II - UNE NECESSAIRE INTERVENTION}

En dépit d'une étonnante précocité économique liée aux échanges maritimes, la Méditerranée des temps modernes est entrée dans une aire de stagnation économique et sociale dont elle émerge avec peine et de manière inégale. Mais, aux lentes évolutions, a succédé soudain un cours précipité du temps. Il s'exprime dans des paysages déchirés dont les éléments anciens sont remployés pour d'improbables perspectives. Tous les âges s'y côtoient désormais. La soudaineté et la brutalité de la modernisation ont des effets dévastateurs, parfois sur les ressources, notamment l'eau douce mais aussi sur les paysages souvent mis en péril.

Les modifications intervenues récemment dans nos relations à la nature ont fait émerger la notion de paysage dans le champ des préoccupations des citoyens avant même qu'elle ne soit devenue un concept scientifique. L'élaboration d'une charte du paysage méditerranéen est apparue alors comme un premier pas vers une prise en charge politique de la question. Elle ne doit pas seulement répondre à une demande sociale mais stimuler les recherches sur cet objet complexe qu'est le paysage à la fois réalité objective et représentation subjective. Objet donc, doublement culturel par la construction qu'il représente et par la vision que l'on porte sur lui, le paysage est à la fois un patrimoine et un enjeu. Cette construction est une structure évolutive qu'il n'est pas souhaitable de figer dans son évolution par une protection qui risque de la naturaliser, au sens propre du terme. Il n'en semble pas moins légitime de ne pas laisser agir librement les forces qui peuvent aboutir à sa destruction. La sagesse suggère alors d'en conduire l'évolution en tenant compte de ses spécificités et notamment de celles qui relèvent du patrimoine qu'il représente et de la plus ou moins grande fragilité de ses composantes «naturelles». Dans tous les cas, il est nécessaire d'en avoir une connaissance préalable approfondie. On peut donc retenir pour examen les trois rubriques suivantes:

- la fragilité des équilibres écologiques sur lesquels reposent les paysages méditerranéens.

- la soudaineté des formes de modernisation qui s'abattent sur ce paysage sous l'effet de techniques ou d'échelles de valeurs extérieures à la Méditerranée.

- on peut y ajouter, si l'on est optimiste, une manière originale d'apprivoiser une nature difficile qui constitue désormais plus un legs qu'une réalité mais qui peut demeurer une source d'inspiration.

\section{III - LA FRAGILITE DES EQUILIBRES ECOLOGIQUES}

La part des composantes «naturelles» du paysage doit être relativisée parce qu'elle prend place dans des systèmes dont la valeur est donnée par les hommes. Ainsi, le climat de la côte d'Azur, prisé au XIX ${ }^{\mathrm{e}}$ siècle par une classe sociale privilégiée pour la douceur de ses hivers, est recherché désormais par un plus grand nombre pour la villégiature estivale. A ces deux visions différentes des caractères 
d'un même climat a correspondu la mise en place de deux paysages différents, la relégation des plus anciens et le remploi du paysage intermédiaire par le dernier en date.

Il n'en demeure pas moins nécessaire de prendre en compte les éléments «naturels» du paysage méditerranéen dans la mesure où les équilibres écologiques, particulièrement fragiles, se présentent sous les dehors trompeurs de l'immuabilité. Cette impression qui tient peut être à l'absence de saisons intermédiaires, à la fréquence des arbres toujours verts et à la solide ossature du relief, est une illusion des sens dont un ouvrage des disciples de Pierre Birot fit justice il y a une dizaine d'années sous le titre explicite: «La mobilité des paysages méditerranéens». Une mobilité ponctuée de légendes et de drames qui fait de la scène méditerranéenne un espace à haut risque pour le paysage et les hommes qui le construisent.

\section{1 - LA MOBILITE DU DECOR MINERAL}

On sait depuis quelques années que la théorie de la tectonique des plaques peut être appliquée à la Méditerranée et que les chaînes alpines internes et de son pourtour s'intègrent dans ce mécanisme. La collision des plaques africaine et européenne est à l'origine d'une présence constante de la montagne dans l'aire méditerranéenne dont elle est un élément essentiel du paysage. On lui doit aussi une instabilité accompagnée de séismes et d'éruptions volcaniques. Ce caractère accidenté du relief et l'importance de la néotectonique, ont fait varier le décor depuis l'Antiquité et confèrent une vigueur particulière à l'érosion. Les sols reflètent d'ailleurs fréquemment les interruptions répétées de la pédogenèse dues soit à des reprises d'érosion soit à un enfouissement sous des apports éoliens ou détritiques. Dans un tel contexte, la moindre modification d'un agro-système peut avoir également des répercussions considérables comme on peut d'ailleurs en constater actuellement les effets en de nombreux endroits avec l'abandon des terrasses et banquettes.

\section{2 - LA DUALITE DU REGIME PLUVIOMETRIQUE}

Le climat méditerranéen fait se succéder dans l'année deux types contrastés de régimes hydriques: la saison froide, durant laquelle se produisent les précipitations propres au régime des pays tempérés océaniques et la saison chaude et aride qui accompagne les hautes pressions tropicales. L'existence d'une saison où coïncident chaleur et aridité est une singularité climatique majeure qui définit le climat méditerranéen; mais sa durée, qui varie dans une année entre un et sept mois, introduit plus que des nuances entre les rivages nord et sud de la Méditerranée. La végétation est affrontée à la double contrainte du froid des hivers et de la sécheresse des étés. Il n'est pas jusqu'aux prairies d'altitude qui ne se ressentent de la sécheresse et où la prédominance des espèces steppiques leur vaut une moindre valeur fourragère que celle des prairies alpines. La forêt à feuilles xérophiles toujours vertes se régénère mal en cas de destruction suivie de pâturage, elle peut alors céder la place à des formations dégradées et discontinues qui laissent les sols sans protection, exposés à une érosion d'autant plus agressive que l'importance des pentes s'ajoute à la violence des précipitations. Par ailleurs, l'irrégularité 
interannuelle des précipitations augmente à l'approche du plus grand désert du monde, introduisant dans les systèmes agricoles traditionnels un risque d'irrégularité de la production. L'effet de ce régime climatique sur les sols en place est un lessivage généralisé et une teneur en humus particulièrement faible. Cela a souvent conduit, en l'absence d'éléments fertilisants en quantité suffisante, à la mise en place de systèmes à longues jachères et retardé d'autant la célèbre «révolution agricole» amorcée au XVII ${ }^{\mathrm{e}}$ siècle dans l'Europe océanique.

\section{3 - LA DEFICIENCE DE L'ECOULEMENT SUPERFICIEL}

L'image des cours d'eau réduits durant l'été à un mince filet courant parmi les galets d'un lit majeur démesuré, est devenue classique; celle des plaines marécageuses également. Et, plus encore peut être, la vision dramatique de ces ravineaux parcourus par une crue soudaine et dévastatrice. Les hommes se sont toujours efforcés de remédier à ces dangers dont la mythologie s'est fait l'écho. Mais les alternances historiques de mise en valeur et d'abandon des plaines littorales disent assez la précarité des conquêtes et la difficulté de trancher une fois pour toute les têtes de l'hydre de Lerne, ces fièvres des aires marécageuses qui ne furent vaincues définitivement qu'au lendemain de la seconde guerre mondiale. La vision «du pays où fleurit l'oranger», celle des plages ou des beaux paysages dont rêvent les gens du nord, a pour effet de masquer des composantes «naturelles» qu'il n'est pas toujours facile de maîtriser et où la moindre erreur peut causer des dégâts irréversibles.

\section{IV - LA COLLISION DES CULTURES ET DES TEMPS}

Si les hommes savent de mieux en mieux s'affranchir des contraintes naturelles, ils deviennent de plus en plus dépendants de mécanismes économiques qu'ils ne savent plus contrôler. Les effets de ce grand désarroi sont partout sensibles y compris sur les paysages méditerranéens pour au moins trois raisons majeures.

- Le maintien tardif des archaïsmes a rendu plus brutaux les changements intervenus depuis un quart de siècle: exode rural, transition démographique, mondialisation de l'économie, etc.

- L'impact de ces changements est souvent gros de dégradation écologique pour des paysages dont on connaît la fragilité.

- Enfin, et bien qu'à des degrés divers, une situation générale de dépendance économique s'accompagne du primat accordé à la vision des gens d'ailleurs c'est à dire celle d'un paysage classique, de loisir culturel et physique, point de vue qui fait bon marché de celui des habitants, attachés à leur cadre de vie et à leur identité culturelle.

\section{1 - LE DEVELOPPEMENT ECONOMIQUE ET SES EFFETS}

Les Etats riverains de la Méditerranée n'ont pas été laissés pour compte en ce domaine. Selon la Banque Mondiale, le produit national brut per capita y accusa, de 1965 à 1983, une croissance annuelle moyenne supérieure à celle de l'ensemble du monde. Cette dernière fut, en effet, de 1, $5 \%$, la même qu'aux Etats-Unis et 
sensiblement la même que la Suisse (1, $4 \%$ ) ou le Royaume-Uni (1,7\%). Par contre la Tunisie, l'Egypte, la Syrie, l'Algérie, le Portugal et la Grèce dépassèrent les $3 \%$, l'Italie, la Turquie et l'Espagne les $2 \%$ et le pays le plus mal placé, le Maroc, se trouvait pourtant largement au-dessus de la moyenne mondiale $(1,8 \%)$. Bien entendu, il faut tenir compte du maintien d'écarts considérables entre les rivages nord et sud en chiffres absolus et du fait que l'accroissement du P.N.B. est d'autant plus important que le point de départ est plus bas. Ces réserves admises, il n'en demeure pas moins que les Etats riverains de la Méditerranée ont connu un développement considérable au cours des trente dernières années.

Une part des revenus est d'ailleurs venue du travail de la population émigrée à l'étranger, ou des dépenses des étrangers venus passer leurs vacances dans ces pays. D'où de profondes modifications dans la répartition de la population mais aussi dans les comportements alimentaires, démographiques, sociaux dont on évoquera quelques unes.

\section{a) Soudaineté et massiveté des changements}

Les pays développés de l'Europe ont connu eux aussi, en leur temps, de tels changements. C'est leur caractère, soudain et brutal à la fois, qui en constitue cette fois l'originalité.

- L'exode rural a eu pour effet de faire passer très rapidement une masse considérable de la population des campagnes vers les villes amenant, dans les Etats les moins peuplés des rivages nord, une véritable dépopulation des campagnes.

- Le tourisme a conduit à un transfert saisonnier massif des pays plus septentrionaux: France, Allemagne, Royaume Uni, etc, vers les plages de la Méditerranée y suscitant une urbanisation considérable et transformant, en quelques années seulement, des bourgades de pêcheurs en centres de villégiature trépidants.

- L'urbanisation massive est partout l'aboutissement de cette évolution, notamment le long des littoraux et s'accompagne aussi de l'apparition et de l'extension des résidences secondaires.

\section{b) Les effets sur les paysages}

Dans les campagnes les plus touchées par l'exode rural, la dépopulation a pu conduire à la déprise du sol et à l'abandon des façons culturales. Les effets sont divers: retour de la forêt mais avec des risques accrus d'incendies périodiques à cause des broussailles, écroulement des murettes sur les pentes et risque de reprise d'érosion. Les paysages peuvent être alors à jamais ruinés ou bien sont réincorporés dans un tout autre contexte. Ainsi, les forêts claires de chênes verts dont la taille périodique n'est plus effectuée peuvent péricliter; à moins d'être entretenues sous forme de parcs de loisir. C'est souvent le cas en bordure d'un lac de barrage, lieu de prédilection des résidences secondaires. Par contre, là où, ponctuellement, le sol se trouve valorisé par une agriculture intensive, l'exploitation s'accompagne d'une consommation d'eau croissante qui peut rapidement conduire à la surexploitation des aquifères voire à leur dégradation irréversible par intrusion d'eaux marines ou 
de rejets polluants. Même des paysages classés tels que les zones humides peuvent se trouver déstabilisés sous l'effet de l'évolution de la zone qui les entoure. La situation n'est pas moins grave dans les espaces urbanisés (fréquence de la pollution atmosphérique, difficultés des transports, spéculation foncière, etc). On connaît notamment l'importance des constructions illicites dans la banlieue de Lisbonne, à Rome et au Caire. Ces perturbations conduisent à des paysages éclatés et transitoires qui comportent des fragments encore fonctionnels d'époques passées. D'où un sentiment de malaise et de désordre et des dysfonctionnements qui peuvent aller jusqu'à retentir sur l'identité des habitants.

\section{2 - LA DIVERSITE DES REGARDS PORTES SUR LES PAYSAGES MEDITERRANEENS}

La Méditerranée a contribué à susciter l'idée de paysage bien que la notion, originaire de l'Europe du nord, lui soit étrangère au départ. C'est que, désormais, le paysage méditerranéen se vend bien. Les ingrédients en sont simples: soleil garanti, mer proche, indigènes menant une vie simple et frugale et même, si nécessaire, quelques beaux vestiges antiques pour ceux qui veulent paraître épris de culture.

En retour, les émigrés qui quittèrent leur pays pour trimer durement dans les usines et chantiers de l'Europe industrielle, aspirent, l'âge venu, à une retraite confortable près de leur lieu de naissance en affichant la modernité qui signe leur réussite sociale. Et le paysage méditerranéen n'est le plus souvent, et de plus en plus, que la juxtaposition des effets de cette vision croisée.

\section{a) L'exaltation sélective du passé}

Quand, en 1833, le royaume de Grèce fut attribué à un prince d'origine étrangère, les souvenirs de l'Antiquité hellénistique furent convoqués pour le choix d'Athènes, la nouvelle capitale et pour le style néo-classique des bâtiments officiels. Il est significatif que cet hommage fut moins le fait des Grecs eux-mêmes que celui d'une monarchie et d'une administration d'origine étrangère. Il devait vite rencontrer, il est vrai, l'acquiescement des «élites» locales au point que ces dernières, dans leur volonté de renouer avec l'Antiquité, en vinrent à négliger l'héritage artistique du passé ottoman. Cette forme perverse d'acculturation exprime bien une certaine idée du paysage classique qui s'est généralisée encore avec le tourisme de masse, grand consommateur d'images simples et valorisantes et de prières sur l'Acropole pour classes moyennes.

\section{b) L'inclusion d'une modernité empruntée}

Une autre forme d'acculturation revient aux maisons d'émigrés de retour au pays. L'effet d'ostentation s'y accompagne d'une inspiration qui emprunte des traits inattendus aux maisons des pays d'émigration et s'accompagne d'exigences coûteuses auprès de municipalités aux budgets étriqués. Chalets alpins ou pastiches médiévaux peuvent ainsi être entourés de jardins soigneusement tondus et agrémentés de statuettes représentant des personnages de Walt Disney et constituer les quartiers neufs de villages de régions reculées et en voie de désertification. On 
trouve ainsi, au Portugal, d'extraordinaires palais du facteur Cheval dans des régions où les loups hurlent toujours et se reproduisent encore!

c) Vers un syncrétisme paysager méditerranéen?

Un des cas les plus étonnants de cette collision chaotique des temps et des cultures, est donné par le village andalou de Palmar de Troya, sur la commune d'Utrera, à une cinquantaine de kilomètres au sud de Séville. Le nom du lieu-dit associe celui d'une formation végétale caractéristique des terres incultes de cette région: celle des palmiers nains (Chamaerops humilis L.) et celui, de consonance antique, d'un grand domaine agricole. Une voie pécuaire importante passait par là, dotée d'un relarguier, sorte d'élargissement de la draille, qui, avec la présence de deux puits permettait aux troupeaux transhumants de s'abreuver et de se reposer. Au total un espace d'environ 500 mètres de long sur 150 de large, se terminant au sud en forme d'entonnoir. Compte tenu du régime social traditionnellement en vigueur dans ces campagnes où de grands domaines employaient une main d'œuvre occasionnelle une brève partie de l'année, les journaliers en quête de travail furent attirés, peu de temps avant la guerre civile, par la construction d'un petit barrage sur le rio Salado le bien nommé. Comme les troupeaux qui empruntaient la draille se faisaient rares et que le terrain de la voie pécuaire relevait de l'Etat et non pas d'un grand propriétaire, la halte servit d'abord de dépôt pour les buses de ciment des futurs canaux d'irrigation. En fait, fermées aux deux extrémités par des couvertures, elles constituèrent les premiers éléments d'habitat auxquels s'ajoutèrent bientôt quelques huttes de branchage. Brülées pendant la guerre civile par le corps franc des grands propriétaires dirigé par le commandant Castejón, les huttes furent à nouveau édifiées en grand nombre et l'un des premiers habitants traça lui même quatre alignements qui devinrent les premières rues du village. En 1960 le village sans nom comptait déjà près de 2.500 habitants mais restait ignoré de l'administration. La population y vivait misérablement dans des huttes rectangulaires de torchis couvertes de branchages. Le cas n'était pas exceptionnel alors dans cette région. A partir du début des années 60, l'émigration temporaire vers l'étranger: l'Allemagne, la France et la Suisse principalement, apporta des ressources qui ne tardèrent pas à retentir sur l'aspect du village. Les maisons en dur se multiplièrent avec les fers à béton de l'espoir dressés vers le ciel et qui promettaient l'adjonction d'un étage. Les maisons à étage suivirent effectivement, puis les commerces et, en dernier lieu, la prise en charge administrative et la construction d'écoles. Une des premières décisions de l'administration franquiste fut d'ailleurs de donner à la rue principale le nom du commandant Castejón en place de «rue des bains» qui évoquait les énormes flaques dont elle était ponctuée. Parmi les éléments du paysage seule la forme du village, aux rues parallèles et se terminant au sud par une longue rue étroite, témoigne de ses origines étranges et pourtant reflet exact d'une organisation sociale bien caractérisée. Le village s'est coulé dans le moule légué par une organisation périmée de l'élevage. L'allure et le style des maisons se ressent pour sa part de cette longue période où les hommes devaient s'expatrier pour faire vivre décemment leur famille. Depuis quelques 
années le village ressemble de plus en plus à d'autres villages andalous et a rejoint une sorte de modèle culturel régional. Il n'est pas jusqu'à la présence incongrue et très apparente d'une secte qui a établi son siège à Palmar qui ne confère à cette nouvelle bourgade l'empreinte d'une modernité douteuse.

d) Le paysage méditerranéen entre les nantis et les déshérités.

Un dernier exemple, pris au Portugal cette fois, permet de poser une question d'ordre plus général. Sur l'espace d'une très grande propriété, à quelques kilomètres à l'ouest de Faro, un immense complexe de loisirs à été édifié avec marina, nombreux hôtels de luxe, terrains de golf, village de vacances, centre commercial, terrain d'aviation et, bien sûr, les indispensables vestiges antiques qui, par chance d'ailleurs, se trouvaient sur place. Vu d'avion cet espace clos par des murs prend l'allure de ce que l'on pourrait appeler «un parc artificiel» mais c'est le contraste avec le paysage environnant qui est, semble-t-il, le plus riche d'enseignement. En bordure de la plage et le long d'un effluent pestilentiel, s'étend, sur trois hectares, le bidonville des Capverdiens venus fournir la main d'œuvre banale nécessaire à l'édification de ce temple de la consommation moderne.

Deux questions ne peuvent manquer d'être posées à l'issue de ce que d'aucuns appellent sans déplaisir «le changement»:

- le paysage étant largement l'expression d'une organisation économique et sociale, l'action sur cette réalité n'est elle pas la façon la plus efficace d'agir sur le paysage?

- l'espace méditerranéen étant pour une large part économiquement dominé, le paysage méditerranéen n'est-il pas traité comme une matière première qui, faute de pouvoir être exportée, peut encore être acquise à bon prix ?

\section{V - UNE CERTAINE MANIERE D'APPRIVOISER LA NATURE}

Les hommes de la Méditerranée surent composer ingénieusement avec des éléments naturels difficiles à contrôler. La frugalité de l'alimentation a fait le reste. Le blé, l'huile d'olive et le vin constituent toujours la base d'une nourriture dans laquelle la valeur énergétique de l'huile d'olive représente un net avantage. D'où l'emprise légère de l'agriculture sèche méditerranéenne qui s'accompagne même souvent d'une sensation de solitude. Par contre, les sociétés méditerranéennes ont jeté très tôt leurs filets sur une nature ingrate, sous forme d'un réseau serré de villes et de bourgades. Elles ont marqué le paysage méditerranéen d'une empreinte indélébile, reflet non seulement d'une organisation économique et sociale mais encore d'une manière d'être, éthos urbain dont le paysage méditerranéen demeure comme imprégné. 
a) Des paysages agraires peu fréquentés

L'étendue des campagnes inanimées ne tient pas seulement à l'étendue des sols squelettiques, à la roche qui partout crève un maigre sol, mais aussi, fréquemment, à une mise en valeur d'intensité décroissante à partir des noyaux de peuplement qui vaut à la coalescence de la périphérie des finages ces paysages désolés et grandioses d'où l'homme parait absent que l'on trouve en Sicile, dans la plaine andalouse et les plateaux d'Alentejo, d'Anatolie ou du Maghreb.

L'emprise agricole sur la partie la plus soigneusement cultivée est elle-même restée longtemps modeste. L'usage de l'araire dans les terres sèches s'est maintenu généralement jusqu'au début des années 60 et la place de la jachère est restée importante jusque là. Cette dernière n'a reculé qu'avec l'introduction de plantes industrielles comme le tournesol ou le coton.

b) Le maintien prolongé des formes traditionnelles d'adaptation

Les formes d'adaptation aux conditions rigoureuses du climat sont demeurées longtemps les mêmes, simples et efficaces bien que peu productives.

L'élevage transhumant du petit bétail ou le nomadisme ont permis d'associer des régions dont les ressources fourragères se complétaient dans l'année. Ces formes d'élevage jouèrent un rôle considérable dans l'histoire économique et politique. Elles ont laissé des traces sous forme de drailles mais aussi des paysages de champs nus ou arborés en forêts claires de chênes verts ou de chênes à vallonées.

L'arboriculture fut pour les agriculteurs une des meilleures façons d'atténuer les aléas climatiques. Les racines des arbres permettant, en allant chercher l'eau en profondeur, d'assurer une certaine régularisation interannuelle de la production. On comprend que l'olivier fut l'arbre sacré par excellence et que la coupe des arbres fut, durant des millénaires, l'opération guerrière la plus redoutée. L'arbre est demeuré un élément majeur du paysage méditerranéen qu'il résulte d'un aménagement forestier comme la forêt de chênes verts ou «dehesa» de la péninsule Ibérique, de plantations et d'améliorations d'arbres propres à la zone comme l'olivier, le figuier ou le caroubier ou encore d'arbres acclimatés comme l'amandier. L'arboriculture fut la providence des agriculteurs refoulés vers les montagnes par les éleveurs et c'est elle qui a permis de nourrir les fortes densités de population de la Kabylie, de la montagne libanaise, des chaînes bétiques, etc. L'arboriculture qui s'est développée sur des pentes fortes s'accompagne alors d'aménagements en terrasses dont l'entretien a exigé un considérable et séculaire labeur dont l'interruption entraîne rapidement la dégradation de ce paysage. L'arbre est enfin l'ultime recours productif lorsque l'agriculture atteint ses limites extrêmes. Que ce soit du côté du désert comme c'est le cas des olivettes du Sahel tunisien entre Kairouan et Sfax ou, plus récemment, sur les sols squelettiques des montagnes schisteuses de la Péninsule Ibérique voués aux plantations d'eucalyptus.

L'irrigation fut la manière la plus efficace de mise en valeur puisqu'elle permet d'oblitérer la plus contraignante des conditions de l'agriculture et de tirer 
pleinement parti de la chaleur et de l'ensoleillement. Pratiquée par les paysans le long des vallées et sur des étendues réduites, très souvent dans les montagnes, avec une ingéniosité étonnante, elle fut le plus souvent le moyen de garantir la régularité des récoltes céréalières. L'essentiel des paysages d'irrigation appartient aux villes qui en conditionnèrent le développement.

\section{2 - L'EMPREINTE DE LA VILLE DANS LE PAYSAGE}

Qui dit ville dit échanges commerciaux et culturels et c'est peut être à ce niveau qu'apparaît le mieux la spécificité par excellence de la Méditerranée et de son paysage. Doublement enfermés dans leurs maisons et dans leurs villes, les hommes de la Méditerranée ont, en fait, fabriqué un des paysages parmi les plus artificiels (au sens étymologique du terme) qui soient au monde. Le comble du raffinement étant constitué, en Italie notamment, par l'intervention du paysage dans la peinture et du peintre dans le paysage. Ce fut le cas avec Benozzo Gozzoli et Domenico Veneziano. A la limite, le paysage méditerranéen est alors devenu une œuvre d'art. La précocité des échanges à grande distance a implanté ici des phénomènes considérés ailleurs comme modernes tels que les spécialisations régionales des productions. L'intensification de l'agriculture induite par ce phénomène s'est manifestée par l'extension de l'irrigation et par son étroite association à la ville. Il n'est pas jusqu'à l'élevage extensif qui n'ait reflété la puissance du patriciat urbain comme ce fut le cas dans des villes de la péninsule Ibérique telles que Saragosse ou Séville, sièges de puissantes associations d'éleveurs.

a) La précocité des échanges maritimes et commerciaux

A partir du troisième millénaire av. J.-C. les techniques nautiques ont rendu possible des échanges, limités d'abord à de courtes distances. Dès lors la Méditerranée fut ce que BRAUDEL a désigné sous le terme «d'espace-mouvement». «La Méditerranée, ce sont des routes de mer et de terre, liées ensemble, des routes, autant dire des villes, les plus modestes, les moyennes et les plus grandes se tenant toutes par la main («La Méditerranée, l'espace et l'histoire», Paris, 1977).

C'est ce commerce maritime qui devait rendre possible des spécialisations régionales qui apparurent dès l'Antiquité et dont les plus importantes furent associées à l'alimentation de Rome, en blé, en huile, en garum et en vin. Il est significatif que le petit mont Testaccio, à Rome, soit constitué des débris d'amphores portant les marques de potiers des rives du Bétis. Cette tradition fut maintenue au Moyen-Age, notamment pour le sucre de canne dont lî̀le de Chypre puis la région valencienne furent les principaux fournisseurs de Venise et de Gênes. La tradition de l'agriculture commercialisée se retrouve ainsi d'un bout à l'autre de la Méditerranée et trouve souvent en elle même les ressources pour se perpétuer sous d'autres formes d'activités. 
b) La tradition des jardins d'eaux

La bourgeoisie urbaine a toujours apprécié des jardins débouchant en Italie, au cours des $\mathrm{XV}^{\mathrm{e}}$ et $\mathrm{XVI}^{\mathrm{e}}$ siècle, sur la notion de «beau paysage». Il diffère d'ailleurs sensiblement dans les deux régions emblématiques que sont, à cet égard, la Toscane et la Vénétie, en fonction des conditions différentes d'acquisition des terres par les nouvelles classes sociales urbaines.

L'eau constitue un élément essentiel dans l'aménagement des jardins à la fois parce qu'elle permet le développement d'une végétation luxuriante, qu'elle se prête à des jeux décoratifs et, enfin, parce qu'elle apporte une fraîcheur appréciée. C'est pour leur plaisir que les bourgeois musulmans de Valence aménagèrent leurs «ruzafas» vers le $\mathrm{XI}^{\mathrm{e}}$ siècle ou que les bourgeois marseillais développèrent autour de leurs bastides, dans la seconde moitié du XIX ${ }^{\mathrm{e}}$ siècle, des jardins d'eau dont il reste encore quelques exemples. Ces derniers firent payer à la ville de Marseille l'embellissement de leurs jardins en arguant de l'augmentation des productions attendue de l'extension de l'irrigation. Christian Tamisier a dénoncé l'hypocrisie du raisonnement mais il n'en demeure pas moins qu'une des formes les plus courantes d'agriculture irriguée fut développée en relation étroite avec l'aménagement des jardins des patriciats urbains. La ghouta de Damas en est une bonne illustration. Par ailleurs, ces jardins d'agrément furent souvent un lieu d'acclimatation de plantes autrefois exotiques et désormais parfaitement intégrées, comme les agrumes, dans le paysage irrigué des «huertas» méditerranéennes. Ces jardins fonctionnèrent alors comme des pépinières permettant un relais dans le temps des productions commerciales. Ainsi, à Valence, lorsque la concurrence de Madère d'abord, de l'Amérique ensuite, ruina la culture de la canne à sucre, le mûrier et l'élevage du ver à soie purent aisément s'y substituer pour céder leur place, dans le courant du $\mathrm{XIX}^{\mathrm{e}}$ siècle, à l'oranger après la maladie du ver à soie. A chaque «cycle» le paysage change mais demeure chargé d'inventivité et marqué du sceau de l'activité commerciale. On pourrait parler de constantes paysagères.

\section{c) Le triomphe contemporain de la ville}

L'extension de l'irrigation demeure la meilleure forme de mise en valeur de la terre mais elle s'exerce désormais de manière exclusive en fonction du marché et donc des villes. Dans ces conditions, il se produit une véritable inversion des paysages. On trouve un bel exemple en Grèce où la plaine de Thessalie, hier encore parcourue par les Valaques et leurs troupeaux, est désormais mise en irrigation dans sa quasi totalité dans le même temps où les montagnes du Pinde, soigneusement mise en valeur par un aménagement séculaire de terrasses et de rigoles d'arrosage, sont en voie de dépeuplement. Désormais l'irrigation peut surgir à peu près partout, soit à partir de forages, soit à partir de transferts hydriques à grande distance et ce ne sont plus les conditions de la géographie physique qui en commandent la répartition mais bien les rapports aux marchés pour écouler les productions et aux capitaux pour assurer les investissements, c'est à dire aux villes.

Même si les conditions nouvelles qui prévalent entraînent une inversion des paysages, c'est bien le triomphe de la ville qui est ainsi consacré dans le droit fil 
d'une tradition. De même, le morcellement du paysage et sa diversité, filles de ses composantes «naturelles» au départ, le sont désormais au regard de la valeur ajoutée.

Tout indique le début d'une nouvelle ère pour le paysage méditerranéen. L'emprise humaine qui demandait peu à l'agriculture et davantage au négoce laisse place à une emprise ponctuelle mais très forte à côté d'une déprise généralisée. Par ailleurs, une modernisation soudaine, d'origine exogène pour une bonne part, s'abat sur un paysage qui prend à son tour une valeur marchande et se dévalorise à mesure qu'il se vend.

\section{CONCLUSION}

La question de la spécificité du paysage méditerranéen peut paraître incongrue tant la réponse semble évidente avec la sécheresse estivale, la lumière, la montagne, la mer enfin qui met en relation les régions bordières et puis, dans l'esprit des hommes, une certaine nostalgie d'un paysage classique authentifiant une qualité de vie et de civilisation que l'on sait désormais fragiles. Il faudrait dire d'abord la singularité du paysage méditerranéen, même s'il a beaucoup emprunté ailleurs, sa diversité, sa mobilité en dépit de ses formidables décors de pierre qui semblent défier le temps, sa fragilité enfin. On notera à ce propos que la fragilité n'est plus seulement écologique, que le morcellement est de plus en plus fondé sur l'économie et que la spécificité majeure qu'il faut prendre en compte c'est que cette construction millénaire que constitue le paysage méditerranéen est devenue l'enjeu de convoitises alors même qu'elle est partie intégrante de notre patrimoine.

S'il y a beau temps que les hommes ont conscience de l'évolution des paysages, le sentiment que se produit actuellement une mutation nouvelle par sa rapidité et sa massiveté est tout récent et comporte la prise de conscience d'un risque. On veut donc connaître les périls pour les mieux conjurer. Dans cette tâche qui rejoint une préoccupation citoyenne, la géographie a un rôle aussi important à jouer que celui qui fut le sien dans l'exploration de la terre, à condition, il est vrai, de ne pas prêter la main à des opérations de fabrication et de vente de paysage méditerranéen qui constituent un des plus sûrs moyens de le détruire ou de s'engager dans un pseudo combat contre «l'imposture écologiste» qui permet, en fait, de conforter d'abord la posture productiviste toujours en vogue.

Compte tenu de la polysémie de la notion de paysage, il apparaît sage de ne le définir qu'en fonction d'un projet qui serait une sorte de code de bonne conduite. Pour y parvenir on se doit de prendre en compte la distinction qui fonde l'irréductible dualité de la notion de paysage. D'un côté un certain nombre de formes et d'objets qui relèvent d'une réalité objective c'est à dire indépendante de l'observateur. De l'autre la perception que les individus ou les communautés ont de ces formes et de ces objets, la représentation qu'ils s'en font et la valeur qu'ils lui attribuent. La résolution de ce qui n'est peut être qu'une contradiction apparente pourrait être cherchée dans la transposition au paysage, singulièrement dans le monde méditerranéen, de ce qu'un de ses fils: André Gide en l'occurrence, proposait pour chaque homme: «suivre sa pente, pourvu que ce soit en montant». 
Appliqué au paysage cela pourrait signifier qu'il convient de le laisser respirer, vivre, évoluer donc, mais dans le cadre de son génie c'est à dire, en l'occurrence, de ses spécificités. 